\title{
CDSWS: coverage-guaranteed distributed sleep/ wake scheduling for wireless sensor networks
}

\author{
Guofang Nan ${ }^{1 *}$, Guanxiong Shi', Zhifei Mao ${ }^{1}$ and Minqiang $\mathrm{Li}^{2}$
}

\begin{abstract}
Minimizing the energy consumption of battery-powered sensors is an essential consideration in sensor network applications, and sleep/wake scheduling mechanism has been proved to an efficient approach to handling this issue. In this article, a coverage-guaranteed distributed sleep/wake scheduling scheme is presented with the purpose of prolonging network lifetime while guaranteeing network coverage. Our scheme divides sensor nodes into clusters based on sensing coverage metrics and allows more than one node in each cluster to keep active simultaneously via a dynamic node selection mechanism. Further, a dynamic refusal scheme is presented to overcome the deadlock problem during cluster merging process, which has not been specially investigated before. The simulation results illustrate that CDSWS outperforms some other existed algorithms in terms of coverage guarantee, algorithm efficiency and energy conservation.
\end{abstract}

\section{Introduction}

With the advances in digital signal processing, RF techniques and low-power hardware manufacturing and integration, wireless sensor networks (WSNs) have attracted increasing interests in recent years [1]. A WSN is structured with a certain number of tiny sensor devices, and each device has the abilities of computation, storage, and communication, which enable it to collect sensing data and conduct data processing tasks about the environment, and to generate and deliver helpful information on the monitored objects to the base station for decision making [2]. The appearance of WSNs has significantly changed various kinds of remote sensing applications such as environmental and ecological monitoring of natural habitats, smart homes, and military areas [3].

In order to provide high-quality data service, a multilevel of sensing coverage and network connectivity is needed in the practical implementation of a WSN. That is, any point in the region should be covered by more than one sensor. Therefore, wireless sensors are usually densely deployed on the target field [4], that is, many sensors can detect an event, deliver and receive the sensed data packets simultaneously, which will cause redundant communication overhead and thereby leads

\footnotetext{
* Correspondence: gfnan@tju.edu.cn

${ }^{1}$ Institute of Systems Engineering, Tianjin University, Tianjin 300072, China Full list of author information is available at the end of the article
}

to large amount of energy consumption. Due to the facts that wireless sensors are physically small and must use extremely limited power or energy, the network lifetime is an essential consideration in sensor network applications. Moreover, a WSN is usually deployed in hostile fields or under harsh environments [5] where manually recharging batteries for sensors is not feasible, one typical alternative approach to energy saving is to turn off some sensors and activate only a necessary set of sensors while providing a good sensing coverage and network connectivity simultaneously [6]. A good sleep/ wake scheduling has to provide an even distribution of energy consumption among sensor nodes so that the network lifetime is extended [7].

Several schemes have been proposed in the literature to determine how many and which nodes should be allowed to sleep [7], and they can be divided into distributed sleep/wake scheduling schemes [8-11] and centralized sleep/wake scheduling mechanisms [12-16]. Generally, centralized sleep/wake scheduling algorithms are appropriate only for stationary targets or moving targets with known and static movement patterns [17], and it is easy to achieve more precise scheduling results. However, for an unknown and dynamically changing movement environment, the centralized sleep/wake scheduling algorithms are not flexible enough to adapt themselves to these changes. Another drawback for the centralized ones is that sleeping nodes should have the ability to 
receive messages all the time, and their receiving antenna cannot be switched off. In addition, a powerful base station [14] is used for centralized scheduling to perform a large amount of computation and communication tasks, and it is dificult for the base station to maintain the global information of the whole network, which will lead to a large amount of data transmission, and thereby cause more energy consumption. On the other hand, most of these distributed sleep/wake scheduling schemes make the sensors self-organized to carry out network tasks, which have less messaging cost and better adaptability to dynamic conditions [16]. Moreover, distributed algorithms are scalable and can work independently for a long time.

However, there are still several major limitations in prior distributed sleep/wake scheduling algorithms. First, it is inconvenient for sensors to maintain sensing coverage and connectivity of the entire network by using these distributed algorithms due to the fact that only local information is used for sensors to decide their status. For example, in [8], an adaptive partitioning scheme called connectivity-based partition approach (CPA) was presented for sleep scheduling and topology control in WSNs, CPA divides the network into several groups, only one node in each group will be selected to be active to form a backbone network. Since the communication radius of sensors is applied in group partitioning stage, the proposed algorithm ensures the effective connectivity of the network, but does not consider the problem of sensing coverage. A geographical adaptive fidelity (GAF) algorithm [18] partitions the nodes into multiple equalsize squared cells based on their geographic locations, and one node in each cell remains active, GAF also ensures network connectivity, but ignores sensing coverage. The authors in [6] also developed a distributed adaptive sleep scheduling algorithm (DASSA) for WSNs with partial coverage, which suits only for temperature or humidity monitoring. Second, most of the distributed algorithms $[6,8,14,17,19,20]$ assume that only one node in each cluster or group is active while others are shut off, which is usually effective in early stage of the network lifetime, and once some nodes are failed to sense and communicate, this mechanism may lead to poor quality of service (QoS) of the network. Third, the deadlock problem arising from resource contention has received little attention in the past, which will result in degraded network performance in distributed sleep/wake scheduling. A deadlock is a persistent and circular-wait condition in forming a cluster or a group [8], where each potential cluster head delivers a merging request message to another cluster, and may involve in a deadlock waiting indefinitely for the merging respond from other nodes while not answering other merging requests [21].
Motivated by above limitations, a novel distributed coverage-guaranteed sleep/wake scheduling algorithm called CDSWS is proposed in this article. In CDSWS, a cluster hierarchy based network framework is considered, and a minimum number of nodes are selected to be active to monitor the area while maintaining better coverage and connectivity in this article. We assume in this article that communication radius of a sensor is equal to or greater than twice of its sensing radius, which has been proved that the coverage of a region implies connectivity of the network [22]. Moreover, a sensor is selected to be in sleep mode based on its sensing radius. That is, if a sensor is in the sleep mode, its whole working area can also be covered by other active nodes, which does not affect whole coverage performance. Thus, any point in the region can be covered by those active nodes and any two active nodes are connected. In addition, a dynamic node selection mechanism is also adopted in each cluster to maintain network performance. Unlike prior work, more sensors in each cluster are allowed to be active simultaneously. Finally, in order to overcome the deadlock problem in clusters merging, a set of rules are illustrated to avoid existing deadlocks. For each clusterhead, when it sends request to other clusters while receiving other requests simultaneously, obtaining respond from its requesting object or answering other requests is determined by these rules, consequently, merging delay and energy consumption are reduced.

The rest of this article is organized as follows. Section 2 gives a brief literature overview. We introduce the motivation and present our solution in Section 3. In Section 4, we propose our coverage-guaranteed scheduling framework and the corresponding algorithms to support our scheduling framework. In Section 5, we present simulation and experiment results to demonstrate the efficiency of the work and compare it with other scheduling techniques. Finally, the advantages and disadvantages of the proposed scheme are discussed in Section 6.

\section{Literature review}

Almost all the literature treat the objective of sleep/wake scheduling as minimizing energy consumption or maximizing sensor network lifetime [23]. However, they make quite different assumptions regarding the sensors and the sensor network, and also propose different approaches in their applications. These approaches can be divided into centralized and decentralized scheduling, deterministic and random scheduling, layer-based scheduling (MAC layer, routing layer, application layer). In this section, we will summary the recent sleep/wake scheduling algorithms. 
Turning off some nodes in the network and using only a necessary set of nodes for information collection and packet delivery is one popular way of energy conservation [16]. GAF uses geographic location information to divide a sensing region into equal-sized grid cells, and each cell of the grid is square shaped, only one node is active in each grid, then forms a backbone network to maintain connectivity [21]. In [24], a few nodes are selected as coordinators which remain active for packet routing, and other nodes go into the sleep state according to a sleep/ wake cycle specified by the coordinators. In [25], a node decides to go into sleep mode if there is an active neighbor within its sensing range, and its sleeping period is self-adjusted dynamically. Otherwise, it remains active. However, this method does not need the location information. The mechanism that randomly selected idle sensors to go into the sleep mode is allowed in the scheduling [26] to save energy. The data packets for sleeping nodes are temporarily stored at the active nodes in their neighbors, and the sleeping sensors wake up periodically to retrieve the stored packets from their neighboring nodes. This method usually leads to packet delay. An adaptive partitioning scheme of sensor networks for node scheduling and topology control was presented in [8] to reduce energy consumption. Sensors are partitioned into several groups according to the measured connectivity between pair-wise nodes, which varies prior partitioning approaches based on sensor locations. In each group, only one node is active while others are put into sleep mode. The authors formulated a constrained optimal graph partition problem to study sleep/wake scheduling with topology control. A distributed heuristic approach called CPA was proposed. The authors in [6] also developed a DASSA for WSNs with partial coverage, which does not require location information of sensors while maintaining network connectivity and satisfying a user defined coverage requirement. A common character among above scheduling schemes is that these active nodes form a backbone network to assure network connectivity without considering sensing coverage.

Some coverage-preserving scheduling algorithms were discussed in [17,27-29]. In [27], each node in the network autonomously and periodically makes decisions on whether to turn on or turn off itself only depending on its local neighbor information. To preserve sensing coverage, a node will turn it off when other active neighbors can help it to cover its whole working area. Optimal CoveragePreserving Scheme (OCoPS) [28] extends the center angles calculation method described by [27], based on the proposal of a wake-up strategy, a new decision algorithm is illustrated to decide the node status by exchanging local information. Aiming at dynamic point coverage, a scheduling algorithm based on learning automata is proposed in [17], the advantage is that less auxiliary messages are needed to be delivered between nodes, and each node in the network is equipped with a set of learning automata which determine when and which node should be in active or asleep state according to environmental information. Experimental results show that the proposed scheme outperforms the existing methods. A coverage-adaptive random sensor scheduling [29] was also presented to meet the desired sensing coverage specified by the users. However, the above methods pay little attention to network connectivity.

The Sense-Sleep Tree (SS-Tree) [10] uses flow models and mathematical programming to the network in accordance with the classification tree structure to solve sleep scheduling. It uses the tree structure of the network scheduling and graph theory was applied to form SS-Tree, the method has a high computing complexity and cannot work in the complex situation. The authors in [12] investigated the cross-layer sleep/wake scheduling design in service-oriented WSNs, the purpose of this study is to minimize the energy consumption and guarantee that enough sensors are active to provide all required network performances. The sleep scheduling is considered to be NP-hard, and a heuristic linear programming based solution is also presented. However, they assume that each service has a known requirement on the number of active sensors based on the historical service composition requests in the system, which may not be the case in practice.

Some centralized scheduling approaches have been investigated. A cluster-based hierarchical network was considered in [14], in this structure, sleep/wake scheduling problem was illustrated based on multi-hop communication. Unlike prior work, this article considered the effect of synchronization error in their sleep/wake scheduling algorithm. Most of computation tasks are performed in a base station which uses the sub-gradient method and computes the capture probability thresholds, then tells the sensor nodes and the nodes decide the wake-up schedule themselves. A centralized sleep scheduling algorithm based on integer linear programming was presented in [6], which calculates the lifetime using the global information of the whole network based on the assumption that the global knowledge of sensor locations and energies is known. According to their proposed scheme, sensors allowed to sleep can be intermittently inactive to reduce energy consumption and thus extend network lifetime. In [30], the authors assumed that all sensors were supplied with approximately the same amount of initial energy and studied the coefficient of variation of energy consumption of three different sleep scheduling schemes: the randomized scheduling (RS) scheme, the distance-based scheduling (DS) scheme, and the Balanced energy Scheduling (BS) scheme. The proposed algorithm is also performed by a cluster head. 
More accurate scheduling results will be achieved by suing the centralized approaches, which also lead to a large amount of data transmission and computation.

Another fundamental issue is the deadlock problem in distributed computing and several deadlock avoiding mechanisms for sensor network applications have been illustrated in the prior literature. However, the aforementioned mechanisms were designed for distributed edge-coloring [31], determining d-dominating sets for coverage tier [32] and secure data aggregation [33]. One possible deadlock scenario will occur for sensors to selforganize into clusters or groups. However, little attention has been given to the deadlock problem in sleep/ wake scheduling.

\section{Motivation and major considerations}

In this section, we analyze several limitations of the existing studies that motivate us to make the nodes selforganize into clusters in pure distributed manner. We also give the main strategies to overcome these limitations.

\subsection{Motivation}

In CPA, sensors are partitioned into several groups based on their measured connectivity between nodes instead of their location information. The process of CPA starts from the initial partition where each node forms a unique group, and two groups are continuously merged into a larger one. CPA has more flexibility because it can achieve $k$-connectivity of the backbone network and switch node status within each group after the partitioning process is finished. Meanwhile, constrained optimal graph partition problem is used to formulate group partitioning, and a distributed implementation of CPA is illustrated. However, it still suffers from several limitations.

First, sensing coverage and network connectivity are two important issues that considerably influence the QoS of an entire network system. However, CPA was designed with the purpose of maintaining the network connectivity, and the sensing coverage was not taken into consideration. The lack of coverage guarantee will clearly render the network more prone to node failures and produce more coverage holes, which will lead to poor network monitoring performance.

Second, in the group merging process of CPA, a priority value is assigned for each two completely adjacent groups, which is given by priority $=k_{1}(1-\alpha)+k_{2} \beta+R$, where $k_{1}$ and $k_{2}$ are coefficients, $\alpha$ indicates the level of equivalence between these two groups, $\beta$ is the ratio of energy in these two groups to the total energy of the entire network, $R$ is a random value uniformly distributed in $[0,1]$. As a result, the appropriate assignment enables pairwise groups with a lower priority value to merge first. It is noteworthy that the calculation of the total energy has to rely on a centralized scheme of information collection that is apparently run contrary to the concept of distributed computation. In practice, numerous data relays among nodes are required to figure out the total energy of the network, thereby increasing the execution complexity. Apart from the execution complexity, the data arrived at sensor nodes tend to be outof-date due to the severe network delay, so the priority value that obtained may be imprecise.

Third, severe deadlocks potentially exist during the process of group merging in CPA. For instance, a request circle will occur among three groups when each group sends simultaneously a merging request to its next group in the circle as shown in Figure 1, and each group do not answer the merging request from its last group. Thus, a deadlock is generated and no further merging process will continue. Generally, a time-out mechanism or a multi-round technique can be exploited to address deadlock problems. However, these approaches will incur undesirable resources abuse in terms of time delay and energy consumption.

Finally, in the startup phase of nodes scheduling in $\mathrm{CPA}$, a large communication over-head will be produced because each node in one group broadcasts a message to announce its inclination to be the head node, which will results in not only energy inefficiency but transmission interferences among sensor nodes. Besides, the node with the maximum residual energy will be selected as the head theoretically by setting the time delay for each node inversely proportional to its residual energy. However, it is usually not the case in practice because of the network delay and poor signal quality.

In our scheme, we modify the group merging constraints and priority formulation, and then introduce a dynamic unlocking method in conjunction with a novel nodes scheduling scheme. In consequence, substantial

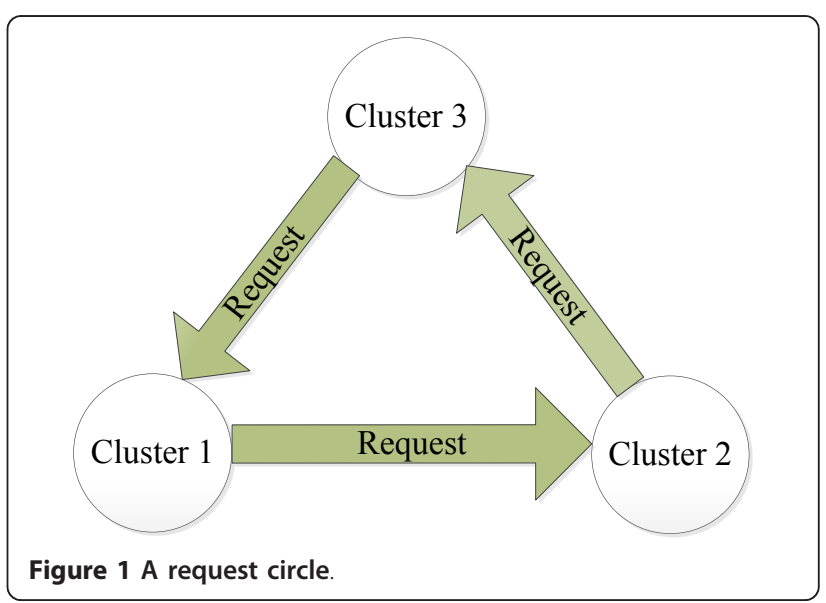


improvement will be achieved in terms of coverage support and algorithm efficiency.

\subsection{Sensing coverage guarantee}

Our CDSWS partition sensors into several clusters based on their sensing coverage rather than measured connectivity. Since it has been proven that if the radio range of the sensor is equal to or greater than twice the sensing range, i.e., $r_{c} \geq 2 r_{s}$, complete sensing coverage implies network connectivity [22]. In other words, in case of $r_{c} \geq 2 r_{s}$, we only need to consider the sensing coverage.

Consider that the sensor nodes are divided into multiple clusters. At any given time, only a few sensors are selected from different clusters to be in active status, while others are put into sleep mode to conserve the precious energy. To maintain a nearly full coverage, the area that can be monitored by the active sensors should be almost equal to the area that can be monitored by all sensors. Hence, the coverage disks of these active sensors should intersect one another within each cluster. However, if two neighboring clusters are not densely connected, they cannot be merged into a larger one.

In terms of minimizing the number of sensors used in deployment, an efficient method to cover the monitored region is to deploy sensors in a triangular pattern with $\sqrt{3} r_{s}$ as the length of a side [34], that is, if the distance between each two nodes within the same cluster is no more than $\sqrt{3} r_{s}$, their coverage disks will be densely connected.

In CDSWS, the concept of neighboring clusters is defined as follows:

Definition 1 (Neighboring clusters): Let $A$ and $B$ be two different clusters, for any node $x_{i}$ in $A$ and any node $x_{j}$ in $B, A$ and $B$ are said to be neighboring clusters if

$$
d\left(x_{i}, x_{j}\right) \leq \sqrt{3} r_{s}
$$

where, $d\left(x_{i}, x_{j}\right)$ denotes the distance between $x_{i}$ and $x_{j}$.

Denote that the numbers of sensors in $A$ and $B$ are $N_{A}$ and $N_{B}$. For any $x_{i} \in A$ and $x_{j} \in B$, if $d\left(x_{i}, x_{j}\right) \leq \sqrt{3} r_{s}$, then the connection value between $x_{i}$ and $x_{j}$ is referred to be 1 , say $c_{i j}=1$. Thereby, the connectivity intensity between clusters $A$ and $B$ can be calculated as

$$
C_{A B}=\sum_{i=1}^{N_{A}} \sum_{j=1}^{N_{B}} \frac{c_{i j}}{N_{A} \times N_{B}}
$$

The calculation of connectivity intensity is executed along with the cluster-forming operation. Clusters $A$ and $B$ can be merged only when $C_{A B}=1$, i.e., $A$ and $B$ are neighboring clusters. Moreover, in order to guarantee the coverage-connectivity in the scheduling process, there must exist at least one other cluster (denoted as $D$ ) that can satisfy $C_{A D}$ and $C_{B D}$ simultaneously. Hence, the connectivity of the area covered by active sensors of these given two clusters $A$ and $B$ can be guaranteed.

\subsection{Priority design in cluster merging}

In cluster merging, each two adjacent clusters that matches sensing coverage requirement is assigned with a utility value. For a given cluster, there may exist several candidate clusters that can be merged with, and only one cluster is allowed to be merged with the given cluster in our algorithm. Therefore, we rank the candidate merging clusters according to their utility value and the one with lower utility value is merged ultimately until no candidate merging clusters satisfy merging requirement.

For a given cluster, several candidate clusters may exist to form different pairwise clusters, if two or more of them have not only the same but the least cluster size. The problem that which one should be merged with the given cluster will become a challenge for the distribute environment. In our algorithm, we introduce a random value distributed in $0[1]$ in the priority formulation to solve this issue. Specifically, to balance energy distribution among all clusters, merging priority should be given to the cluster pairwise with the least sensors. One reason is that it will spend more time for sensor nodes in small-size clusters to be in active status due to periodically alternative working mechanism, which thereby leads to unbalanced energy distribution among clusters. Furthermore, it is convenient for the cluster heads to maintain only the sensor number information of their clusters, thus avoiding collection of the nodes' residual energy which is hard to implement. For any two adjacent clusters $A_{i}$ and $A_{j}$, the number of sensors in them are $\operatorname{size}\left(A_{i}\right)$ and size $\left(A_{j}\right)$, respectively. The priority value is given by

$$
\text { priority }=\operatorname{size}\left(A_{i}\right)+\operatorname{size}\left(A_{j}\right)+R
$$

where, $R$ is a random value uniformly distributed in $[0,1]$.

\subsection{Dynamic refusal scheme}

A purely distributed algorithm is characterized by concurrency which may bring deadlocks. There are four necessary and sufficient conditions for a deadlock to occur.

(a) Mutual exclusion condition. There is a kind of resource that cannot be used by more than one process at a time. In the cluster merging process, for example, each cluster can simultaneously merge with only one another cluster at most. 
(b) Hold and wait condition. Processes already holding resources may request new resources held by other processes. During cluster merging, each cluster holds a resource (i.e., the cluster itself) and meantime waits for another cluster that holds the same resource to merge with.

(c) No preemption condition. No resources can be forcibly removed from a process that holds it, and resources can be released only by the explicit action of the process. A cluster who broadcasts a merging request would not accept a merging request from other clusters.

(d) Circular wait condition. Two or more processes form a circular chain where each process waits for a resource that the next process in the chain holds. Given that the sensor nodes are homogeneous and the clusters are equal with each other, it is reasonable to suppose that circular waiting does exist during cluster merging.

Considering the severe consequence brought by deadlock, it is necessary to design an approach to break at least one of the four conditions that would produce a deadlock. Here, we introduce a dynamic refusal scheme to combat the deadlock problem through avoiding circular-waiting.

Specifically, each initiative cluster opens a timer and creates a variable named WaitPossibility after delivering the request for merging, the value of which indicates the possibility of accepting the request and refusing other requests. This value is initially set to 0.9 , and then decreases by a fixed rate with time until it reaches zero. During cluster forming process, a cluster that receives a merging request will first check the source of the request rather than directly accept it, if the source is one of its merging targets, the cluster can deliver an acknowledge to accept the request with a possibility of the value of WaitPossibility (particularly, 0 means a refusal).

\subsection{Sleep/wake scheduling}

Due to the fact that sensors within the same cluster are densely deployed, the connectivity of the area covered by active sensors can be guaranteed. It is noteworthy that the selection of active sensors in different clusters is independent from others. Thus, to evaluate the connectivity between the cluster and its neighboring clusters, a connection value for each cluster (denoted as A) is calculated as follow.

$$
\mathrm{CON}_{A}=\sum_{B \in \text { neighbor }(A)} C_{A B}
$$

It can be seen from (4) that the connection value for each cluster is evaluated by the summation of the connectivity intensities between $A$ and its neighboring clusters. In addition, we can see that if $C O N_{A}$ is larger than a given threshold $\eta$, only one sensor with the highest energy is needed to be active. Otherwise, two sensors are required to keep in active mode in cluster $A$.

\section{CDSWS: coverage-guaranteed distributed sleep/ wake scheduling}

We considered the scenario where nodes are densely deployed into a region of interest. That means that only some nodes are selected to be active to maintain sensing coverage and network connectivity. The goal of our CDSWS is to partition these nodes into clusters, and at least one node in each cluster is allowed to be active to perform monitoring task. Meanwhile, nodes in the same clusters work alternatively to save energy. Our CDSWS has three major phases: initialization phase, cluster forming phase and sleep/wake scheduling phase.

In this scheme, each cluster is a basic running unit. First it runs an initiative thread to search any clusters that can be merged with, and then opens a listener for receiving messages from other clusters. The initiative thread can be divided into two parts, i.e., initiative searching and merging. In order to control the distributed process, we specify six states for each cluster to differentiate its working status.

\section{Decision}

A cluster is said to be in Decision state when it has not sent its merging request or has already finished a round of merging process, and this cluster can accept merging requests from other clusters in this state.

\section{Contending}

A cluster that has decided its merging target and has sent the request message will go into this state so that this cluster can deal with the request from other clusters through the dynamic refusal mechanism.

\section{Waiting}

A cluster will be switched into Waiting state immediately after responding an initiative cluster, and wait for a corresponding merging instruction until waiting delay exhausts or receiving a instruction.

\section{Locking}

The cluster will stop searching, namely entering Locking state, while all of its neighboring clusters enter the merging process. Its locking level is determined by the number of lock messages from its neighboring clusters.

\section{Merging}

Two neighboring clusters who reach an agreement to merge with each other will both start the Merging process regardless of other merging requests. 


\section{Disposed}

When two clusters are successfully merged into a new and larger one, their IDs will be disposed from the memory of their respective head nodes and neighbors.

Each cluster switches between these six states in the cluster forming process until the whole process ends. Besides, because the process runs in a distributed manner, it is necessary for the nodes and cluster heads to memorize several important variables of the algorithm, which are shown in Tables 1 and 2 .

\subsection{Initialization phase}

In the initialization phase, sensor nodes transmit and receive packets randomly after they are deployed. Therefore, every node obtains the location information of its neighbors. The proposed algorithm is a distributed heuristic algorithm, it starts from the initial partition where each node forms a unique cluster while being the head itself and opens a listener preparing to receive messages from other clusters. These clusters enter Locking state first, and then broadcast a message to search for neighbors and memorize their IDs while wait responses. Afterwards, the cluster head sends an UPDATE message to its neighbors, which contains the basic information of the cluster. As long as a cluster receives an UPDATE message from each of its neighbors, it goes into Decision state. At the end of the initialization phase, nodes move to the cluster forming phase where two potential clusters will be merged into a large one through a user predefined iterative process until no cluster pairs can be further merged.

\subsection{Cluster forming phase}

Once all the clusters have entered Decision state, each of them creates a thread to run the initiative searching process. Meanwhile, its listener keeps open and waits for messages. The initiative searching process consists of three steps. First, each cluster calculates its merging priority by using the information of its neighbors as mentioned in Section 3. If a cluster finds a target to merge with, it will memorize the ID of the target and enter into Contending state and it will stop the whole merging process. Second, under the former circumstance in step 1 ,
Table 2 Variables in cluster head

\begin{tabular}{ll}
\hline Name & Description \\
\hline ID & Identification of the cluster \\
Head & Head of this cluster \\
Members & Members in the cluster \\
Neighbors & Neighbors of this cluster \\
State & Current state of the cluster \\
WaitPossibility & Possibility of this cluster refusing requests from other \\
MergingTarget & ID of another cluster considered as the merging target \\
LockLevel & Locking level of this cluster \\
\hline
\end{tabular}

the cluster delivers a MERGE_REQ message to the target and opens a timer while the value of WaitPossibility is set to 0.9 which will be decreased over time. Third, the cluster waits for a reply from the target until its state is turned into Disposed. The procedure of initiative searching is summarized in Algorithm 1.

The listener of a cluster works all the time during the whole merging process, and it responses each received message from other clusters. To distinguish the received messages with distinct functions, we design a number of message heads for the listener and their corresponding trigger shown in Algorithms 2, 3, 4, 5 and 6.

\section{MERGE_REQ}

It is a merging request which is sent by an initiative cluster to its merging target.

\section{MERGE_ACK}

It is an acknowledgement of MERGE_REQ. A cluster that receives a MERGE_REQ and in Contending or Waiting state will first check the source (namely ID of this source node) of the message according to the content of its MergingTarget. If the source is one of its merging target, the cluster either deliver a MERGE_ACK to accept the request or refuse it by using the dynamic refusal scheme.

\section{MERGE NAK}

It is a refusal of MERGE_REQ. A cluster that receives a merging request but not in Decision or Contending state will deliver a MERGE_NAK to refuse this request. As to a cluster that receives a MERGE_NAK, its state will transfer into Decision and then initiate next searching.

\section{Table 1 Variables in node}

\begin{tabular}{ll}
\hline Name & Description \\
\hline ID & Identification of the node \\
Location & Geographic location of the node \\
Address & Network address of the node \\
$r_{c}$ & Communication radius of the node \\
$r_{s}$ & Sensing radius of the node \\
IsHead & A Boolean variable indicating whether the node is the head of the cluster \\
MyCluster & ID of the cluster the node belongs to \\
\hline
\end{tabular}




\section{MERGE}

This is a merging rule for two clusters. If a cluster is not in MERGING state, it will check whether the merging message is from its mergingTarget. If yes, the merging begins and both of these clusters first turn their states into Merging, the cluster with more sensor nodes will be the initiative one and its cluster head will record the information of the new merged cluster. After two clusters are merged, they will be in DECISION state.

\section{CANCEL}

It is a cancellation of its former merging request sent by an initiative cluster to its merging target. A cluster in the state of Waiting will reset the content of MergingTarget and go into Decision state when it receives this CANCEL message.

\section{LOCK}

It is a locking signal. Clusters stepping into Merging state, namely starting merging, will broadcast a LOCK message. A cluster that not being in the state of Merging or Waiting will raise its locking level by 1 while receiving one LOCK, and go into the state of Locking while the locking level is high than 0 .

\section{UPDATE}

It carries information of an initiative cluster that has already been merged with another one. When a cluster in the state of Locking receives a UPDATE message, its locking level will decrease by 1 , and it will transfer into Decision state if the lock level is 0 .

\section{DISPOSED}

Its content is the information of a passive cluster that has been merged by another. When the cluster in the state of Locking receives this message, its lock level will decrease by 1 as well as receiving a UPDATE message.

The whole flow diagram of cluster forming is shown in Figure 2.

\subsection{Sleep/wake scheduling phase}

Once the cluster forming process is completed, every cluster starts the process of sleep/wake scheduling. In order to save energy, only one or two nodes with highest residual energy in each cluster are required to keep active, while others turn off their radio devices, i.e., being asleep.

In the continuous data-gathering mechanism, each sensor node in a given cluster works periodically. At the beginning of sleep/wake scheduling, all the nodes have to keep active to configure the network, i.e., active node(s) selection. Generally, node(s) with highest energy will undertake the sensing task in a cluster. The cluster head decides which node(s) should be in active state. In one cluster, the cluster head delivers a WORK message to order the selected node(s) to perform its/their duty as working node(s), moreover, one of which is told to be the head node in the next period. Meanwhile, the head delivers a SLEEP message to all of the rest nodes. All the sleeping nodes will wake up and send a WORK_REQ to the cluster head to participate in node(s) selection when the next round comes. While receiving WORK_REQs from all the sleeping nodes in the cluster, the head will run the process of selecting active nodes. The procedures that deal with distinct messages are summarized in Algorithm 7.

\subsection{Time complexity analysis}

Now we analyze the complexity of CPA and CDSWS. We only analyze cluster or group merging phase because it significantly affects the time connectivity of whole algorithms. Assume that $n$ sensors are deployed into an area of interest, and the average connectivity degree is $m$. In CPA, the connectivity level for every node is firstly measured, and complexity of calculating the connectivity level is $O\left(n^{2}\right)$. Another important phase is the group merging which includes calculating merging priority and ranking. In the process of calculating merging priority, the maximum value of connectivity for each group is $m$. So the complexity of executing union or intersection operation for every two candidate groups is $O\left(\mathrm{~m}^{2}\right)$. It is also required to measure the level of equivalence between these two groups, which can be indicated as a cartesian product of two groups, so the connectivity becomes $O\left(m \times \frac{n}{m}\right) \times O\left(m^{2}\right)$, that is $O\left(n m^{2}\right)$. Further, the ratio of energy in two groups to the total energy of the entire network is then calculated, and the complexity is $O(n+2 m)$. Thus, the complexity of calculating merging priority is approximate to $O\left(n m^{2}\right)$. In the process of sorting merging priority for all candidate merging groups, because the number of groups is uncertain in each iteration and the maximum value is $n$, the complexity can be denoted as $O(n)$. Therefore, in each iteration of the merging and ranking phase, the complexity is $O(n)+O\left(n m^{2}\right)$, and the maximum number of iterations does not exceed n, so the complexity is $O\left(n^{2}\right)+O\left(n^{2} m^{2}\right)$. Therefore, the whole complexity of CPA is $O\left(n^{2}\right)+O\left(n^{2} m^{2}\right)+O\left(n^{2}\right)=$ $O\left(\left(m^{2}+2\right) n^{2}\right)$.

In CDSWS, we only calculate the time complexity for each node because our CDSWS is a distributed scheme. After sensors are initially deployed, each node records information from at most $m$ adjacent nodes, and the complexity is $O(m)$. Unlike CPA, CDSWS only ranks candidate nodes or clusters that satisfy merging conditions, and the number of candidate nodes or clusters does not exceed $m$. Normally, the number of merging requests of nodes is larger than $m$ but smaller than $m^{2}$ because not all the merging requests can be really achieved due to concurrency of nodes. Therefore, the whole complexity of each node in our CDSWS does not exceed $O\left(\mathrm{~m}^{3}\right)$. Actually, the run time of each node may not be fully in accordance with the 


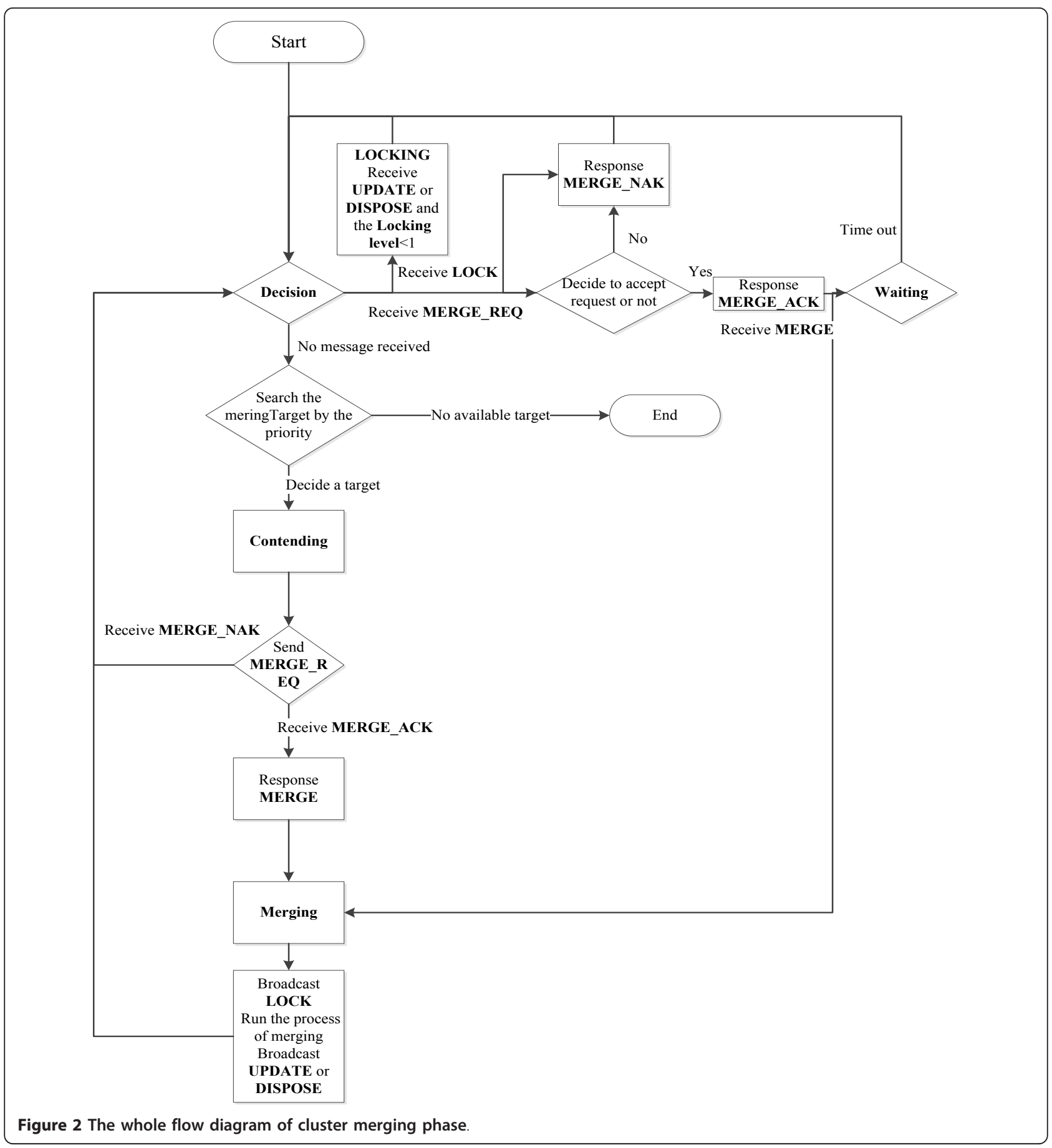

above theoretical analysis due to concurrency of nodes, even so, our CDSWS still performs better than CPA in time complexity.

\section{Performance evaluation}

\subsection{System configuration}

Our algorithm is simulated with homogenous sensors randomly deployed in square areas. Both the communication radius and sensing radius are fixed in each experiment, and the value of which varies in different experiments. It is also assumed that each node has an initial energy of 500 Units and $1 \mathrm{~s}$ of work costs 1 Unit of energy. To simulate the behavior of sensor nodes as in real environment, a $30 \mathrm{~ms}$ delay is added in each communication. 


\subsection{Cluster merging}

The dynamic refusal scheme is a key technique to tackle circular waiting, which further improves the efficiency of clusters forming. In this experiment, we evaluate the impact of dynamic refusal scheme on cluster forming in terms of merging time.

Here, a WSN is con figured with different sensor densities (say 1-5 sensors per grid) randomly deployed in a $100 \times 100$ square area which is divided into 25 grids with the size of $20 \times 20$ for each. In order to compare the performance of our algorithm with that of CPA, the communication radius and sensing radius are set equal to those in CPA, say $40 \sqrt{5}$ and $20 \sqrt{5}$, respectively. To reduce the effects of stochastic factors involved in the test, we run this experiment 30 times and then average the results.

Figure 3 shows that the merging efficiency is significantly improved by using the dynamic refusal scheme, especially for the deployments with higher node density. For example, approximately $30 \mathrm{~s}$ is reduced with the deployment of five nodes per grid. Figure 4 shows that the merging time cost per node decreases to around $0.25 \mathrm{~s}$ by a $0.1 \mathrm{~s}$ from nearly $0.35 \mathrm{~s}$ with the deployment of one node in each grid, and even $0.2 \mathrm{~s}$ is saved when there are five nodes in each grid. With higher node density, both the total merging time and the merging time per node increase almost linearly, which should be attributed to the increasing frequency of merging and information exchanges. However, both of them can be significantly reduced by using our dynamic refusal scheme, this can be interpreted under the scenario where circular waiting will more possibly occur as node density increases, the efficiency of cluster forming will deteriorate due to frequent and unavoidable failures to merging handshaking.

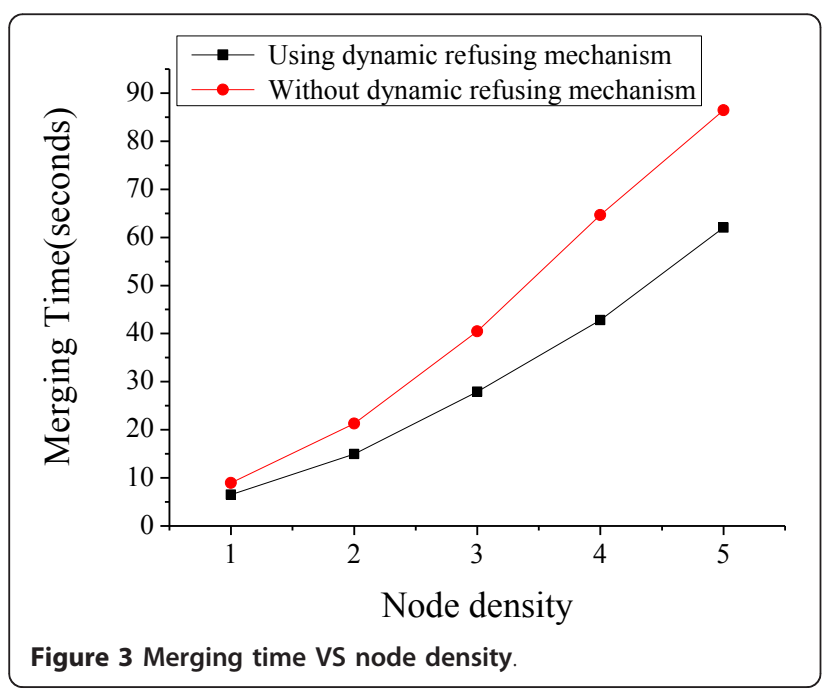

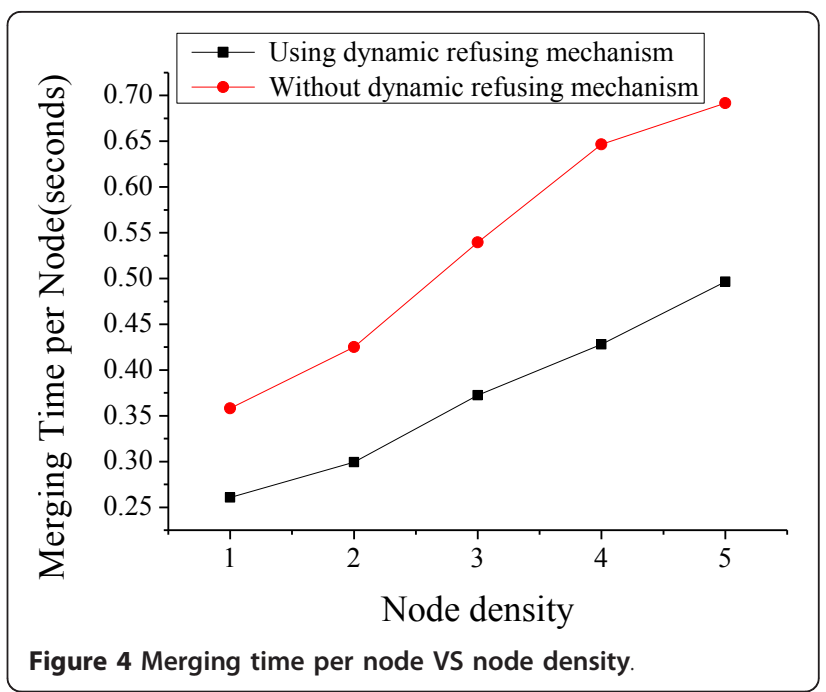

In addition, a comparison of average cluster size is made between CPA and our CDSWS. The table below provides the comparison result when the node density is 5 . Because parameter controls the minimum degree of the 2-induced graph of the final partition in CPA [8], we list four different clustering results with different settings. We notice from Table 3 that the average size of each cluster of our algorithm is larger than that of CPA. This is because the merging constraint in CDSWS is relaxed while CPA does not consider the coverage guarantee. In our CDSWS, the average size of each cluster is larger that that of CPA, which means that less clusters are produced. Moreover, in most cases, the connection value between a cluster and its neighbors is larger that the threshold, only one sensor in each cluster needs to be in active state. Therefore, a minimum number of nodes are selected to be active.

\subsection{Sleep/wake scheduling}

In this section, we investigate the effects of sleep/wake scheduling. The simulation region is a $100 \times 100$ square area which is divided into $10 \times 10$ grids with a grid of $10 \times 10$ for each. The communication radius and sensing radius are set to $40 \sqrt{5}$ and $20 \sqrt{5}$, respectively, as the same in the cluster forming part. First, we compare our algorithm with CPA in terms of the number of

Table 3 Average cluster size produced by CDSWS and CPA

\begin{tabular}{ll}
\hline Partition approach & Average cluster size \\
\hline CDSWS & 9.4 \\
CPA (mindeg $=2)$ & 7.0 \\
CPA (mindeg $=3)$ & 6.0 \\
CPA (mindeg $=4)$ & 5.5 \\
CPA (mindeg $=5)$ & 4.7
\end{tabular}


working nodes in the process of sleeping scheduling. Table 4 shows that the number of working nodes in our algorithm during each working round is less than that in CPA, especially when $\eta=2.4$, it achieves the least working nodes, i.e., only 60 nodes for node density 2 and 64 nodes for node density 3. Furthermore, Table 5 also indicates that the coverage rate reaches more than 99\% with different values of $\eta$.

We also show the impact of $\eta$ on the number of working nodes. In this example, the communication radius and sensing radius are set to 20 and 10 , respectively.

Figure 5 illustrates the general trend of the number of working nodes. With the growth of the number of deployed nodes, the numbers of working nodes experience a sharp increase first, followed by a comparatively steady and marginal rise. But the corresponding twists show a little difference, say around 75 for the $50 \times 50$ area. More importantly, the value of shows a strong correlation with the number of working nodes. That is, more working nodes are required to achieve a higher value of $\eta$. Moreover, because the average size of each cluster in our scheme is larger that that of CPA, the number of working nodes by our CDSWS is thereby smaller that that of CPA.

\subsection{Coverage guarantee}

In this section, we study the coverage issue. Here, the monitoring area is $50 \times 50$ which is divided into 25 grids. The communication radius and the sensing radius are 20 and 10 , respectively.

In Figure 6, we observe that the coverage rate becomes higher with the increasing number of deployed sensors. We also investigate the impact $\eta$ of on sensing coverage in Figure 6. Here, $\eta$ is a threshold that controls how many sensors are selected to be active. When deployed sensors are fixed, $\eta$ larger will lead to more active nodes, which thereby improves the whole sensing coverage. However, $\eta$ has only a small influence on the sensing coverage for the scenario where sensors are densely deployed. In addition, the coverage rates generated by CDSWS and CPA can achieve almost complete coverage when more than 150 nodes are deployed into the region.

Table 4 Number of working nodes needed by using CDSWS and CPA

\begin{tabular}{lll}
\hline Number of working nodes & Destiny $\mathbf{~ 2}$ & Destiny $=\mathbf{3}$ \\
\hline CDSWS $(\eta=2.4)$ & 60 & 64 \\
CDSWS $(\eta=3.0)$ & 69 & 75 \\
CDSWS $(\eta=3.6)$ & 71 & 87 \\
CPA (mindeg $=2)$ & 66 & 70 \\
CPA (mindeg $=3)$ & 76 & 81 \\
CPA (mindeg $=4)$ & 88 & 90 \\
\hline
\end{tabular}

Table 5 Coverage performance evaluation by our CDSWS under different value of $\eta$ and different node-density deployments

\begin{tabular}{llll}
\hline Coverage & $\boldsymbol{\eta}=\mathbf{2 . 4}(\%)$ & $\boldsymbol{\eta}=\mathbf{3 . 0}(\%)$ & $\boldsymbol{\eta}=\mathbf{3 . 6}(\%)$ \\
\hline Destiny $=1$ & 99.575 & 99.753 & 99.920 \\
mDestiny = 2 & 99.827 & 99.895 & 100 \\
Destiny = 3 & 100 & 100 & 100 \\
\hline
\end{tabular}

Another experiment is carried out to capture the effect of $\eta$ on network coverage with the network running. The results are shown in the Figure 7 . In the earlier stage, where the energy of each node is sufficient, the network coverage keeps steady. A higher level and a higher level of coverage is always with a higher value of $\eta$. Then, the figure shows a slight fall in the coverage after a long steady process due to energy consumption. Finally, the network coverage degrades dramatically as the energy consumption is aggravated. In contrary to the earlier stage, a higher value of $\eta$ will result in a lower coverage level. The reason is that more active nodes are required to maintain a higher level of network coverage (denoted by a higher value of $\eta$ ), and nodes will consume energy faster until their failures. Thus, it is impossible to keep a higher level of network coverage by more active nodes. Accordingly, there is an essential tradeoff between coverage guarantee and network lifetime, that is, a higher $\eta$ indicates a better coverage but a shorter network lifetime, and vice versa. Meanwhile, we simulated the trend of the coverage performance of CPA with the network running. It also shows a slight fall and performs worse than CDSWS when $\eta$ is set to 2.4 and 3.0.

\subsection{Network survivability}

Finally, we evaluate our CDSWS from the perspective of network survivability which mainly involves the energy consumption of nodes during their working period.

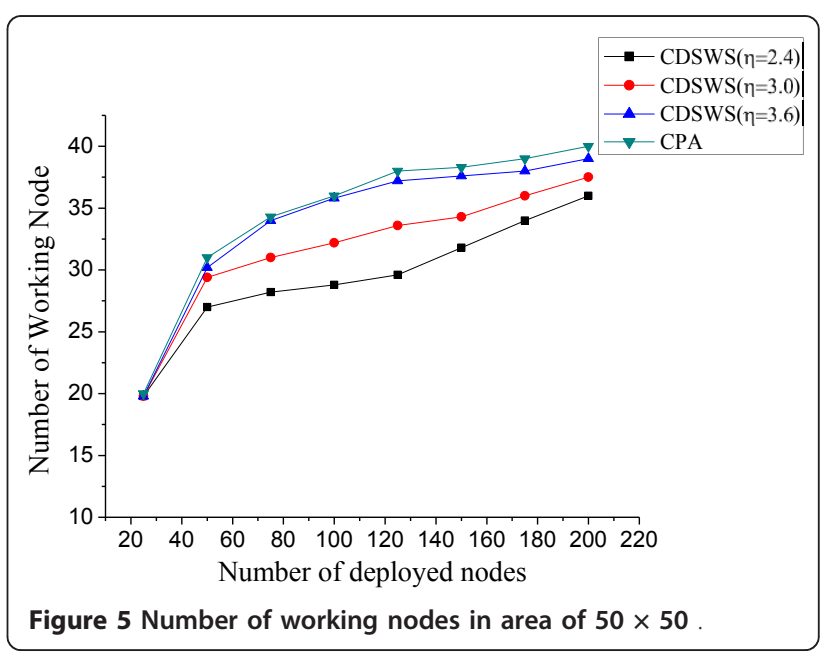




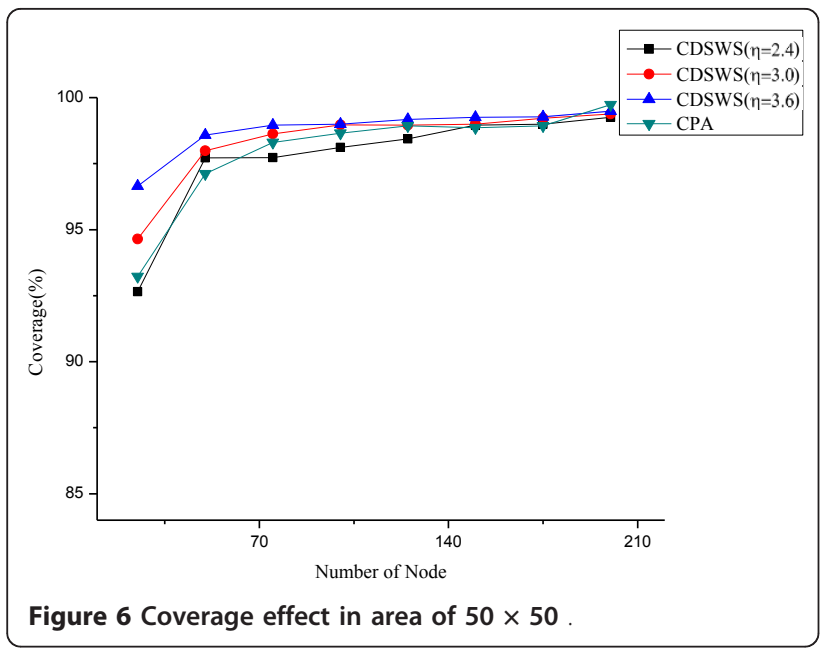

Because energy consumption in cluster forming phase is comparatively low, it is not included in this example.

Figure 8 shows a comparison between CDSWS and CPA in terms of fraction of survival nodes. Apparently, our algorithm has a stronger survivability than CPA, especially at the end of network lifetime since the scheduling by CDSWS makes the energy consumption of nodes in each cluster more balanced and needs fewer working nodes than CPA. In other words, nodes in network with CDSWS have a considerably long survivalcycle.

\section{Conclusion}

This article studied the sleep/wake scheduling problem in WSNs and proposed a new CDSWS to improve network performance. There are three advantages in this algorithm, i.e., coverage guarantee, algorithm efficiency, and energy balance. Coverage is a fundamental QoS of topology control in WSNs, we studied coverage

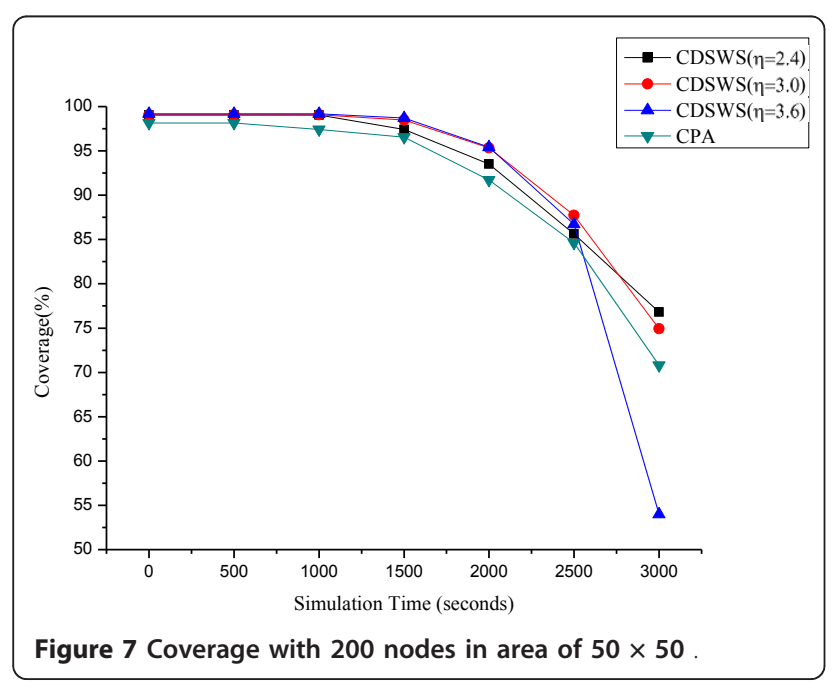

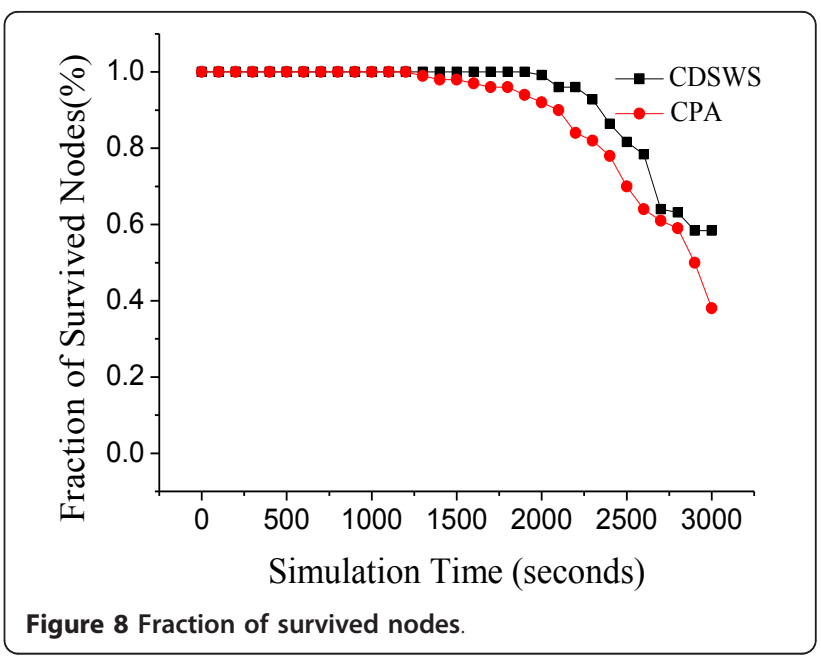

provision based on a prior proof that if the radio range of sensor is equal to or greater than twice the sensing range, then complete coverage implies connectivity, as a result, the network connectivity is provided as well. Second, algorithm efficiency is another essential aspect of our proposed algorithm. Due to the fact that inefficient ones always produce long time delay and energy dissipation, this problem was thereby investigated by avoiding deadlock during cluster forming phase. Accordingly, we designed a dynamic refusal scheme to break circularwaiting by setting a time-varying possibility in each cluster, which determines whether a merging request should be accepted. The last but not least important, we obtained an energy-balanced network by allowing more than one node in each cluster to keep in an active state. Simulations were conducted to evaluate the performance of our proposed algorithm. The results illustrate that CDSWS outperforms existing algorithms with respect to coverage guarantee, algorithm efficiency and energy conservation.

\section{Algorithm 1 - Procedure of initiative searching}

$1:$ for search for the mergingTarget if it exists \{

2: if the state of the cluster is Decision \{

3: $\quad$ set the state of the cluster Contending;

4: $\quad$ if the state of the cluster is not Disposed \{

5 : send MERGE_REQ to mergingTarget;

6: $\quad\}$

7: $\quad$ set WaitPossibility $=0.9$;

8: $\quad$ while the state of the cluster is not Decision \{

9: $\quad$ set the WaitPossibility- $=0.2$ every 100 milliseconds;

10: $\quad$ if the state of the cluster is Disposed

11: return;

12: $\quad\}$

13: $\quad$ set delay with $1000^{*}$ size(cluster) milliseconds;

14: $\quad\}$

15: $\}$ 


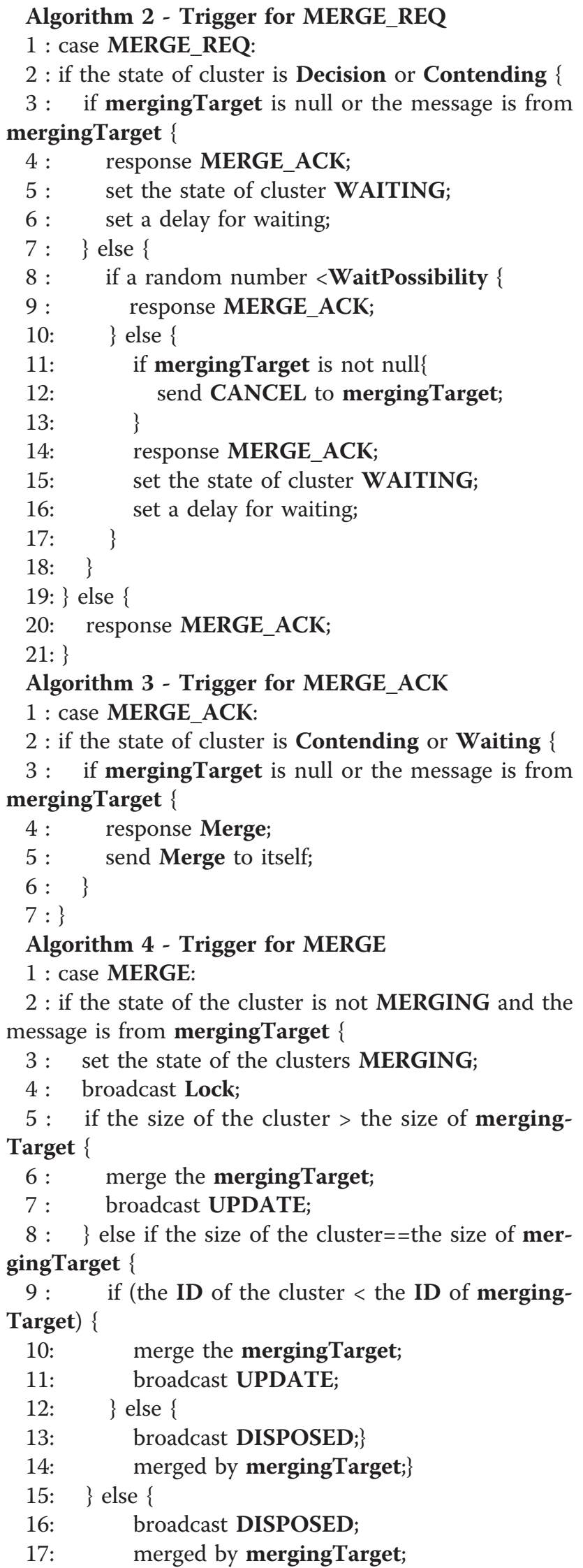

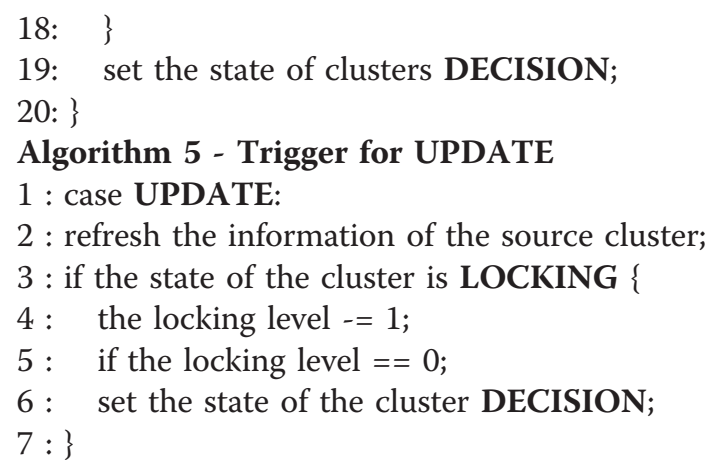

Algorithm 6 - Trigger for DISPOSED

1 : case DISPOSED:

2 : delete the record of the source cluster;

$3:$ if the state of the cluster is LOCKING \{

4: $\quad$ the locking level $-=1$;

5: if the locking level $==0$;

$6: \quad$ set the state of the cluster DECISION;\}

$7:\}$

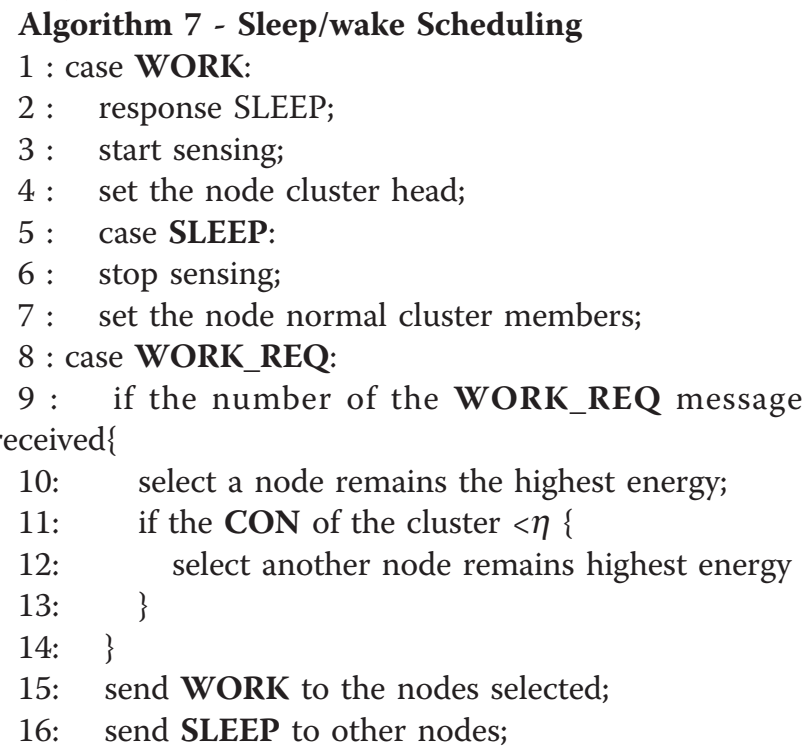

\section{Acknowledgements}

This research is partially supported by research grants from the National Science Foundation of China under Grant Nos. 70701025 and 71071105, the Program for New Century Excellent Talents in Universities of China under Grant No.NCET-08-0396, and a National Science Fund for Distinguished Young Scholars of China under Grant No.70925005, and the Program for Changjiang Scholars and Innovative Research Team in University.

Author details

${ }^{1}$ Institute of Systems Engineering, Tianjin University, Tianjin 300072, China

${ }^{2}$ Department of Information Management and Management Science, Tianjin University, Tianjin 300072, China

Competing interests

The authors declare that they have no competing interests.

Received: 17 November 2011 Accepted: 14 February 2012 Published: 14 February 2012 
References

1. CK Ting, CC Liao, A memetic algorithm for extending wireless sensor network lifetime. Inf Sci. 180(24), 4818-4833 (2010). doi:10.1016/j. ins.2010.08.021

2. GF Nan, MQ Li, Energy-efficient query management scheme for a wireless sensor database system. EURASIP J Wirel Commun Netw. 2010, 1-18 (2010)

3. K Kalpakis, K Dasgupta, P Namjosh, Efficient algorithms for maximum lifetime data gathering and aggregation in wireless sensor networks. Comput Netw. 42(6), 697-716 (2003). doi:10.1016/S1389-1286(03)00212-3

4. H Jun, W Zhao, MH Ammar, EW Zegura, C Lee, Trading latency for energy in densely deployed wireless ad hoc networks using message ferrying. Ad Hoc Netw. 5(4), 444-461 (2007). doi:10.1016/j.adhoc.2006.02.001

5. A Chehri, P Fortier, M Tardif, UWB-based sensor networks for localization in mining environments. Ad Hoc Netw. 7(5), 987-1000 (2009). doi:10.1016/j. adhoc.2008.08.007

6. T Yardibi, E Karasan, A distributed activity scheduling algorithm for wireless sensor networks with partial coverage. Wirel Netw. 16(1), 213-225 (2010). doi:10.1007/s11276-008-0125-2

7. E Bulut, I Korpeoglu, Sleep scheduling with expected common coverage in wireless sensor networks. Wirel Netw. 17(1), 19-40 (2011). doi:10.1007/ s11276-010-0262-2

8. $Y$ Ding, $L$ Wang, $L$ Xiao, An adaptive partitioning scheme for sleep scheduling and topology control in wireless sensor networks. IEEE Trans Parallel Distrib Syst. 20(9), 1352-1365 (2009)

9. JJ Niu, Distributed self-learning scheduling approach for wireless sensor network, in 2nd International Conference on Future Computer and Communication, Wuhan, China, pp. 253-257 (21-24 May 2010)

10. RW Ha, PH Ho, XS Shen, Cross-layer application-specific wireless sensor network design with single-channel csma mac over sense-sleep trees. Comput Commun. 29(17), 3425-3444 (2006). doi:10.1016/j. comcom.2006.01.019

11. B Chen, K Jamieson, H Balakrishnan, R Morris, SPAN: an energy-efficient coordination algorithm for topology maintenance in ad hoc wireless networks. Wirel Netw. 8(5), 481-494 (2002). doi:10.1023/A:1016542229220

12. JP Wang, DY Li, GL Xing, HW Du, Cross-layer sleep scheduling design in service-oriented wireless sensor networks. IEEE Trans Mobile Comput. 9(11), 1622-1633 (2010)

13. NA Pantazis, DJ Vergados, DD Vergados, C Douligeris, Energy efficiency in wireless sensor networks using sleep mode TDMA scheduling. Ad Hoc Netw. 7(2), 322-343 (2009). doi:10.1016/j.adhoc.2008.03.006

14. Y Wu, S Fahmy, NB Shro, Sleep/wake scheduling for multi-hop sensor networks: non-convexity and approximation algorithm. Ad Hoc Netw. 8(7), 681-693 (2010). doi:10.1016/j.adhoc.2010.02.002

15. T Yardibi, E Karasan, A distributed activity scheduling algorithm for wireless sensor networks with partial coverage. Wirel Netw. 16(1), 213-225 (2010). doi:10.1007/s11276-008-0125-2

16. E Bulut, I Korpeoglu, DSSP: a dynamic sleep scheduling protocol for prolonging the lifetime of wireless sensor networks, in 21st International Conference on Advanced Information Networking and Applications Workshops, Niagara Falls, Canada, pp. 725-730 (21-23 May 2007)

17. M Esnaashari, MR Meybodi, A learning automata based scheduling solution to the dynamic point coverage problem in wireless sensor networks. Comput Netw. 54(14), 2410-2438 (2010). doi:10.1016/j.comnet.2010.03.014

18. Y Xu, J Heidemann, D Estrin, Geography-informed energy conservation for Ad Hoc routing, in Proceedings of the 7th Annual International Conference on Mobile Computing and Networking, Rome, Italy, pp. 70-84 (July 2001)

19. M Peng, Y Xiao, PP Wang, Error analysis and Kernel density approach of scheduling sleeping nodes in cluster-based wireless sensor networks. IEEE Trans Veh Technol. 58(9), 5105-5114 (2009)

20. TR Sheltami, E Shakshuki, Neighbor-aware clusterhead with different sleep scheduling protocols, in International Conference on Parallel Processing, Portland, USA, pp. 143-147 (8-12 Sept 2008)

21. YB Ling, SG Chen, CYJ Chiang, On optimal deadlock detection scheduling. IEEE Trans Comput. 55(9), 1178-1187 (2006)

22. XR Wang, GL Xing, CY Zhang, CY Lu, R Pless, C Gill, Integrated coverage and connectivity configuration in wireless sensor networks, in Proceedings of the 1st International Conference on Embedded Networked Sensor Systems, Los Angeles, USA, pp. 28-39 (2003)

23. L Wang, Y Xiao, A survey of energy-efficient scheduling mechanisms in sensor networks. Mobile Netw Appl. 11(5), 723-740 (2006). doi:10.1007/ s11036-006-7798-5
24. B Chen, K Jamieson, H Balakrishnan, R Morris, Span: an energy efficient coordination algorithm for topology maintenance in Ad hoc wireless networks. Wirel Netw. 8(5), 481-494 (2002). doi:10.1023/A:1016542229220

25. $F$ Ye, G Zhong, L Lu, L Zhang, PEAS: a robust energy conserving protocol for long-lived sensor networks, in 10th IEEE International Conference on Network Protocols, Los Angeles, USA, pp. 200-201 (2002)

26. W Ye, J Heidemann, D Estrin, An energy-efficient MAC protocol for wireless sensor networks, in Proceedings of the 21st International Annual Joint Conference of the IEEE Computer and Communications Societies, California, USA, pp. 1567-1576 (2002)

27. D Tian, ND Georganas, A coverage-preserving node scheduling scheme for large wireless sensor networks, in Proceedings of the 1st ACM International Workshop on Wireless Sensor Networks and Applications, Atlanta, USA, pp. 32-41 (2002)

28. A Boukerche, X Fei, RB Araujo, An optimal coverage-preserving scheme for wireless sensor networks based on local information exchange. Comput Commun. 30(14-15), 2708-2720 (2007). doi:10.1016/j.comcom.2007.05.018

29. W Choi, SK Das, Coverage-adaptive random sensor scheduling for application-aware data gathering in wireless sensor networks. Comput Commun. 29(17), 3467-3482 (2006). doi:10.1016/j.comcom.2006.01.033

30. J Deng, YS Han, WB Heinzelman, PK Varshney, Balanced-energy sleep scheduling scheme for high-density cluster-based sensor networks. Comput Commun. 28(14), 1631-1642 (2005). doi:10.1016/j.comcom.2005.02.019

31. S Gandham, M Dawande, R Prakash, Link scheduling in wireless sensor networks: distributed edge-coloring revisited. J Parallel Distrib Comput. 68(8), 1122-1134 (2008). doi:10.1016/j.jpdc.2007.12.006

32. B Pazand, A Datta, A three-tiered node scheduling scheme for sparse sensing in wireless sensor networks. Comput Commun. 33(3), 350-364 (2010). doi:10.1016/j.comcom.2009.10.003

33. K Wu, D Dreef, B Sun, Y Xiao, Secure data aggregation without persistent cryptographic operations in wireless sensor networks. Ad Hoc Netw. 5(1), 100-111 (2007). doi:10.1016/j.adhoc.2006.05.009

34. HH Zhang, JC Hou, Maintaining sensing coverage and connectivity in large sensor net-works. Ad Hoc Sens Wirel Netw. 1(1-2), 89-124 (2005)

\section{doi:10.1186/1687-1499-2012-44}

Cite this article as: Nan et al:: CDSWS: coverage-guaranteed distributed sleep/wake scheduling for wireless sensor networks. EURASIP Journal on Wireless Communications and Networking 2012 2012:44.

\section{Submit your manuscript to a SpringerOpen ${ }^{\circ}$ journal and benefit from:}

- Convenient online submission

- Rigorous peer review

- Immediate publication on acceptance

- Open access: articles freely available online

- High visibility within the field

- Retaining the copyright to your article

Submit your next manuscript at $\gg$ springeropen.com 